\title{
Peculiarities and asymmetry of polarization reversal in Pt/PZT-film/Pt:Ti/SiO $/$ /Si-substrate structures in pyroelectric response investigations
}

\author{
S.L. Bravina, E. Cattan*, N.V. Morozovsky, D. Remiens* \\ Institute of Physics NASU, 46, prospect Nauky, 03028 Kyiv, Ukraine \\ E-mail: bravina@iop.kiev.ua \\ * IEMN-DOAE- MIMM CNRS-UMR 8520, Universite de Valenciennes et du Hainaut-Cambresis, \\ 9, Le Mont Houy, 59313 Valenciennes Cedex, France \\ E-mail: denis.remiens@univ.valenciennes.fr
}

\begin{abstract}
By RF magnetron sputtering method the Pt/PZT-film/Pt:Ti-sublayer/SiO $/ \mathrm{Si}$-substrate structures were prepared and pyroelectric response amplitude and phase behaviour under external voltage application was investigated by photopyroelectric modulation method.

The results of investigation of pyroelectric response - external voltage loops of polarization reversal, pyroelectric response - voltage poling curves and pyroelectric response - time repolarization curves and also dynamic current-voltage characteristics of $\mathrm{Pt} / \mathrm{PZT} / \mathrm{Pt}: \mathrm{Ti} / \mathrm{SiO}_{2} / \mathrm{Si}$ substrate structures are presented.

From variation of pyroelectric response in the current and voltage modes the capacity-voltage loops of polarization reversal and poling curves were derived. From asymmetric pyroelectric response - time repolarization curves the voltage behaviour of characteristic times of zero response and saturation was analyzed. Observed transformations of current-voltage characteristics display the considerable voltage and time dependent variation of charge transfer conditions.

The performed investigation has shown the strong correlation between the poling pyroelectric and so ferroelectric and electrical asymmetries. Presented data on the polar and time asymmetry of the conditions of polarization reversal are discussed in the terms of influence of dynamics of space charge asymmetry on pinning conditions under the different polarity of applied voltage in the course of polarization reversal.
\end{abstract}

Keywords: PZT-film/Si structures, pyroelectric response hysteresis loops, polar and poling asymmetry.

Paper received 20.05.04; accepted for publication 21.10.04.

\section{Introduction}

Polar PZT ceramics are known as materials with excellent piezoelectric and pyroelectric properties [1,2], which are retained in PZT films [3, 4]. A good technological compatibility with Si-base of modern electronics makes PZT films be a leader in creation of elements of highdensity dynamic random access memories (DRAM's), non-volatile random access memories (NV-RAM's), micro-electro-mechanics (MEM's) and infrared sensorics (IRS's) [3-5].

The practically important electrical characteristics of "metal-PZT-film-metal on Si-substrate" system as those of other systems of such type manifest the well-known set of natural and technological asymmetries connected with specificity of reversing the poling state in the electroded ferroelectric film on the substrate [3-5].

The asymmetries of ferroelectric hysteresis loops and current-voltage characteristics were found not only for strongly asymmetric $\mathrm{Au} / \mathrm{PZT} / \mathrm{Si}$ heterojunction structures [6] and also for weak asymmetric Ni/PZT/Pt/Si structures, which also possess the pyroelectric asymmetry [7].

Pyroelectricity is well known not only as important applicable effect [2] but also as a high informative method of the polar state investigation [8], especially when pyroelectric response amplitude and phase behaviour was investigated by photopyroelectric modulation method [8]. In this method the sample is investigated in the operation modes of pyroelectric detector of radiation (PDR).

In this paper we present the results of investigations of polar and poling asymmetries of the set of pyroelectric 


\section{S.L. Bravina et al.: Peculiarities and asymmetry of polarization reversal in ...}

characteristics of $\mathrm{Pt} / \mathrm{PZT}$-film/Pt:Ti/SiO $/ \mathrm{Si}$-substrate systems obtained by photopyroelectric modulation method.

\section{Experimental}

\subsection{Samples}

The samples of PZT films were prepared by radio frequency magnetron sputtering method on platinized $\mathrm{SiO}_{2} / \mathrm{Si}$ substrate.

The bottom Pt:Ti-bilayer was formed by platinum bottom electrode with a titanium adhesive layer $(150 \mathrm{~nm}$ of $\mathrm{Pt}$ and $10 \mathrm{~nm}$ of Ti) deposited on $350 \mathrm{~nm} \mathrm{SiO}_{2}$ layer on (100) $n$-type Si substrate.

For substrate stabilization the annealing treatment of the Pt: $\mathrm{TiO}_{\mathrm{x}} / \mathrm{SiO}_{2} / \mathrm{Si}$-substrate structure just before of PZT deposition was performed at $350-400^{\circ} \mathrm{C}$. Then PZT film was deposited on the bottom electrode. The sputtering target obtained by uniaxially cold pressing includes the mixture of $\mathrm{PbO}, \mathrm{TiO}_{2}$ and $\mathrm{ZrO}_{2}$ in the stoichiometric composition. The polar perovskite phase of the PZT-film was obtained by post-annealing treatment at $600-650^{\circ} \mathrm{C}$ during $0,5-1$ hour. The top Pt-electrodes were deposited through a shadow mask by sputtering procedure, which was followed by a lift-off, and have $\approx 1 \mathrm{~mm}^{2}$ of area. The details of the sputtering conditions were described elsewhere $[9,10]$.

The obtained PZT-films with Zr/Ti ratio 54/46 are near the morphotropic phase boundary, which corresponds to better performances for bulk PZT ceramics.

The thickness of the main components are the following: $150 \mathrm{~nm}$ for the top Pt-electrode, $1.9 \mu \mathrm{m}$ for layer for PZT layer, $350 \mu \mathrm{m}$ for Si substrate.

Each element has a current-carrying thin stripe with a circle current electrode of $1 \mathrm{~mm}$ of diameter on the stripe end.

\subsection{Measurements}

For the investigations of pyroelectric and ferroelectric characteristics the measuring set for complete pyroelectric and ferroelectric characterization [8] was used.

The measurements of amplitude $U_{\pi}$ and phase $\varphi_{\pi}$ of pyroelectric response were carried out by photopyroelectric modulation method in the current and voltage modes. In the current mode $U_{\pi}=U_{\pi 1} \propto \gamma / c_{1}$, and in the voltage mode $U_{\pi}=U_{\pi 2} \propto \gamma / c_{1} \varepsilon \cdot f_{m}$, (here $\gamma$ is the pyroelectric coefficient, $c_{1}$ is the volume heat capacity, $\varepsilon$ is the dielectric permittivity, $f_{m}$ is the modulation frequency) $[2,8]$. Since $U_{\pi 1} \propto \gamma$ and $U_{\pi 2} \propto \gamma / \varepsilon$, the dielectric ratio $D_{\pi}=U_{\pi 1} / U_{\pi 2} f_{m} \propto \varepsilon_{\pi}$ reflects the behaviour of dielectric permittivity obtained by pyroelectric measurements.

Under examination of loops of pyroelectric response hysteresis $\left(U_{\pi^{-}} V\right.$-loops and $\varphi_{\pi^{-}} V$-loops $)$ the Pt/PZT/Pt:Ti/ Si structure was investigated in the operating mode of ferroelectric bolometer, at that applied d.c. voltage $V_{d c}$ was varied stepwise $\pm(0,25-1) \mathrm{V}$ cyclically in the range of $-10 \mathrm{~V} \leq V_{d c} \leq+10 \mathrm{~V}$. The same mode was used under investigations of variations of transient pyroelectric response in due course under application of d.c. voltage

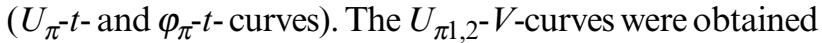
after previous pulse depoling of the samples up to zero response, which remains during a long period without poling voltage application. The $U_{\pi^{-t} \text {-curves were obtained }}$ after previous $11 \mathrm{~V}$ d.c. poling of the samples up to saturated $U_{\pi}$ value, which remains during a long period without poling voltage application.

For minimization of polarization reversal contribution under dynamic current-voltage characterization (I-V-curves) the unipolar saw-tooth drive voltage $V_{d}$ with $0,5 \mathrm{~s}$ of durability and $1 \mathrm{~Hz}$ of repetitive frequency in the amplitude range of $0 \leq V_{d} \leq 10 \mathrm{~V}$ was used.

During pyroelectric measurements the structure under investigation was irradiated by modulated IR-probe from IR LED supplied by generator of sinusoidal voltage through the matching stage [8].

The matching stage for sensitive elements (SE) of PDR, based on FET impedance transformer with using dynamic load and changeable impedance in the input circuit was applied $[11,12]$. It successively operates in the current mode (power measurement) and voltage mode (energy measurement) in the range from infrasound up to low ultrasound frequencies. So, the investigated Pt/PZT/ $\mathrm{Pt}: \mathrm{Ti} / \mathrm{Si}$ structures were placed in the conditions of operation of the real SE of PDR.

\section{Results and comments}

\subsection{Pyroelectric response characteristics}

\subsubsection{Pyroelectric response - modulation frequency characteristics}

The dependences $U_{\pi 1,2}\left(f_{m}\right)$ and $\varphi_{\pi 1,2}\left(f_{m}\right)$, obtained for the investigated samples of Pt/PZT film/Pt:Ti/Si after positive ("+") and negative ("-") d.c. poling are presented in Figures $1 a$ and $1 b$.

The shapes of $U_{\pi 1,2}\left(f_{m}\right)$ and $\varphi_{\pi 1,2}\left(f_{m}\right)$ are near identical for "+" and "-" poled SE (compare Figs $1 a$ and $1 b$ ). Consecutive repolarization of SE gives $180^{\circ}$-addition to $\varphi_{1,2}$ which corresponds to the change of sign of pyroelectric reaction of PZT film and only insignificant variations of shape of $U_{\pi 1,2}\left(f_{m}\right)$ and $\varphi_{\pi 1,2}\left(f_{m}\right)$ dependences. This indicates the almost complete pyroelectric reversibility of reorientation of polarization direction in interelectrode space of Pt/PZT/Pt:Ti structure. A small scatter of $U_{\pi 1,2}$ values can be explained by the difference in the degree of unipolarity of PZT film under the top Pt electrode and the bottom Pt:Ti one.

\subsubsection{Pyroelectric response - voltage poling curves}

Figure $1 c$ presents the dependences of pyroelectric response value on the poling voltages lower than the coer- 


\section{S.L. Bravina et al.: Peculiarities and asymmetry of polarization reversal in ...}
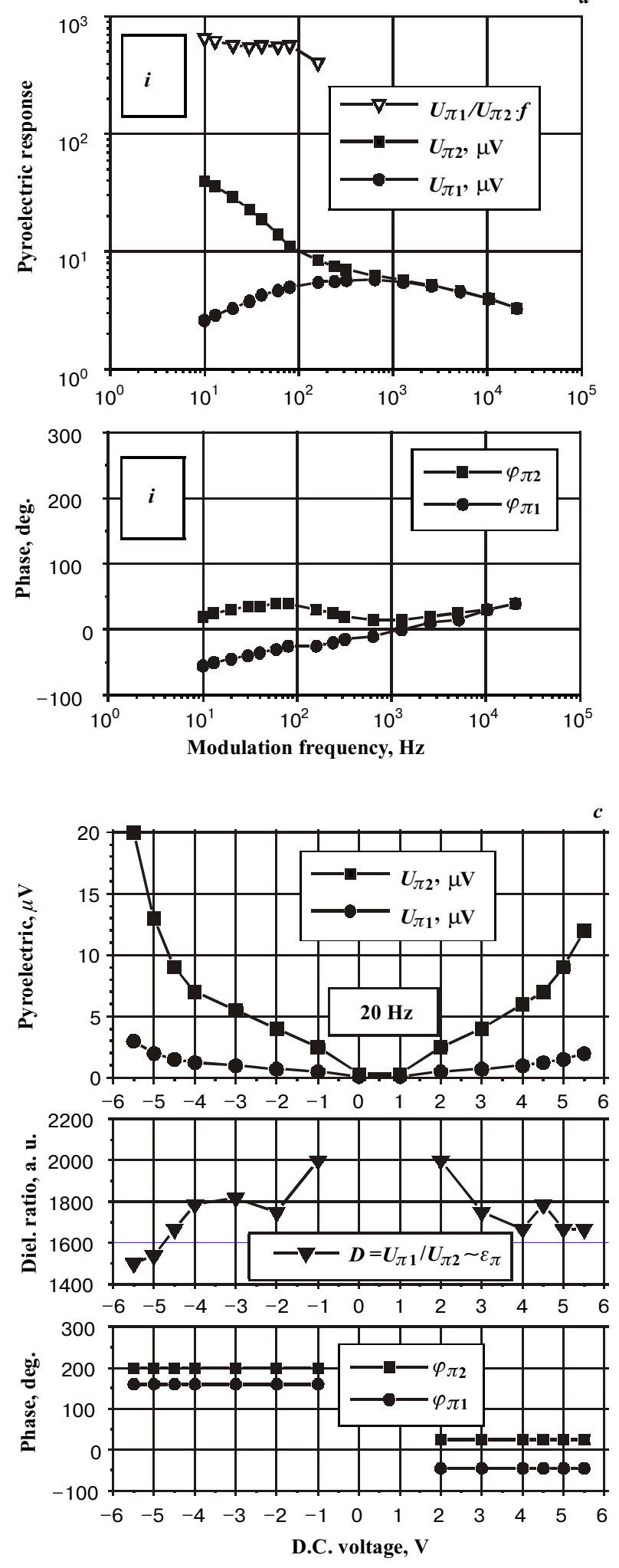

Fig. 1. Modulation frequency dependences $(a, b)$ and poling curves (c) of pyroelectric response amplitudes $U_{\pi 1,2}\left(f_{m}\right)$, dielectric ratio $D_{\pi}=U_{\pi 1} / U_{\pi 2}$ fm and phases $\varphi_{\pi 1,2}\left(f_{m}\right)$ :

$a-$ after $+35 \mathrm{~V}, 5$ min d.c. poling;

$b-$ after $-35 \mathrm{~V}, 5 \mathrm{~min}$ d.c. poling.

$c$ - under d.c. poling after complete depoling.
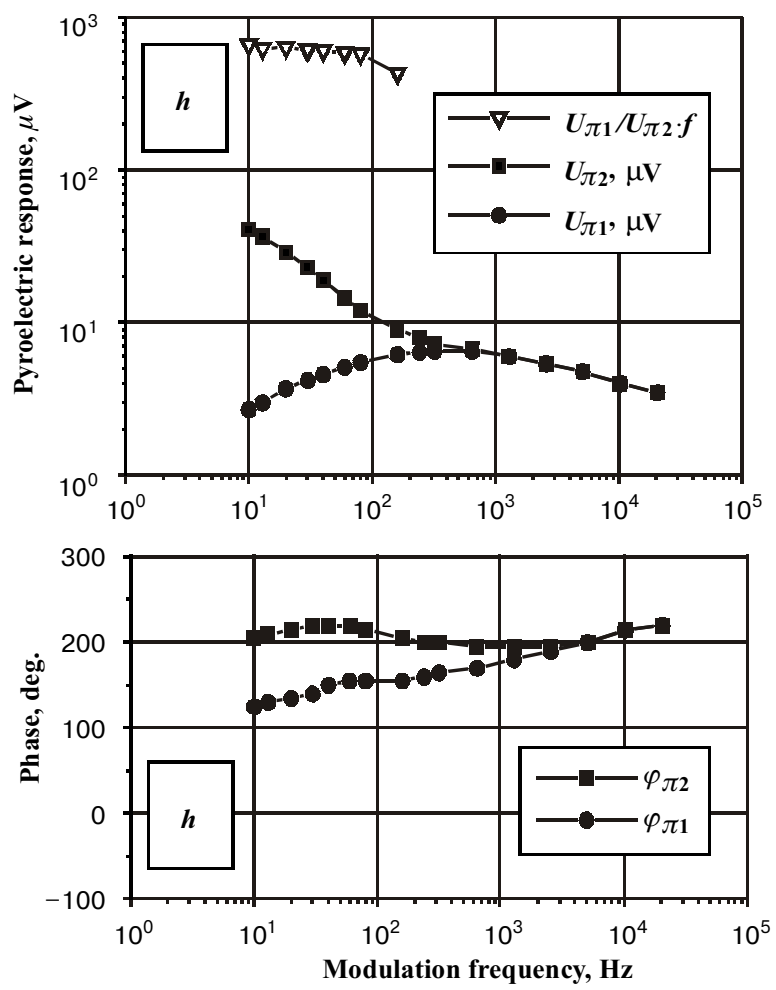

cive ones and also near them. The application of the external d.c. voltage $V_{d c}$ leads to the increase of $U_{\pi 1,2}$ from zero to saturated values. These saturated values increase with the increase of $V_{d c}$ and remain during a long period after getting off the $V_{d c}$ of both negative and positive polarities.

Obtained $U_{\pi 1,2^{-}} V$-curves display the asymmetry of positive and negative branches which consists in particular in positive shift of zero response regions and the breaks in the vicinity of the threshold voltages $V_{t h}{ }^{ \pm}$. Observed threshold inequality of $U_{\pi 1,2^{-}} V$-curves $\left(V_{t h}{ }^{-}<V_{t h}{ }^{+}\right)$corresponds to the coercive inequality of $U_{\pi 1,2^{-}} V$-loops $\left(V_{c}^{-}<\right.$ $<V_{c}^{+}$) (see part 3.1.3).

For observed behaviour of $\varphi_{\pi 1,2^{-}} V$-curves is characteristic $180^{\circ}$-change of $\varphi_{\pi 1,2}$ under change of $V_{d c}$ polarity, which corresponds to the change of the sign of pyroelectric reaction under polarization reversal.

Difference in $V_{t h}{ }^{-}$and $V_{t h}{ }^{+}$values $\left(V_{t h}{ }^{-}<V_{t h}{ }^{+}\right)$are visible under examination of the behavior of dielectric ratio - voltage curves $\left(D_{\pi^{-}}-V\right.$-curves $)$ (Figure $\left.1 c\right)$. Since $D_{\pi}\left(V_{d c}\right)=U_{\pi 1}\left(V_{d c}\right) / U_{\pi 2}\left(V_{d c}\right) \propto \varepsilon_{\pi}\left(V_{d c}\right)$ the $D_{\pi}\left(V_{d c}\right)$ dependency reflects the behavior of $\varepsilon_{\pi}\left(V_{d c}\right)$.

The tendency of changing $D_{\pi}\left(V_{d c}\right)$ observed on $D_{\pi^{-}} V$ curves indicates the decrease of $\varepsilon_{\pi}\left(V_{d c}\right)$ under $V_{d c}$ increase, which corresponds to decrease of the number of domain walls in the film under poling. The peculiarities of positive and negative branches of $D_{\pi^{-}} V$-curves around of $V_{t h}{ }^{ \pm}$are more diffused for $V_{t h}{ }^{-}$than for $V_{t h}{ }^{+}$. This reflects some differences in rearrangements of domain structure of the $\mathrm{Pt} / \mathrm{PZT}$ film/Pt:Ti/SiO $2 / \mathrm{Si}$ systems under different polarities of external voltage. 


\subsubsection{Pyroelectric response - external voltage poling - repoling loops}

In Figures $2 a$ and $2 b$ the obtained loops of pyroelectric response amplitude $\left|U_{\pi 1,2}\left(V_{d c}\right)\right|$ and phase $\varphi_{\pi 1,2}\left(V_{d}\right)$ and also their combination into real signal $U_{\pi 1,2}\left(V_{d c}\right)$ are presented.

The $\left|U_{\pi 1,2}\right|-V$-loops have the typical "butterfly"-like shape, which is often observed for strain-electric field response loops of PZT films [13].

The behaviour of $\left|U_{\pi 1,2}\left(V_{d c}\right)\right|$ is characterized by the regions of saturation at the front of the "wings" where $V_{d}>V_{c}$ and sharp changes with pronounced minima on the "tails" of the "wings" in the vicinity of $V_{d}=V_{c}$.

The $\varphi_{\pi 1,2^{-}} V$-loops have the characteristic parallelogram-like shape with the regions of $\varphi_{\pi 1,2}$-change on $180^{\circ}$ in the vicinity of $V_{d c}=V_{c}$ and $\varphi_{\pi}\left(V_{d c}\right)=$ const outside the range of $V_{d}=V_{c}$.

Symmetry of the front parts of both positive and negative $\left|U_{\pi 1,2}\left(V_{d c}\right)\right|-$ "wings" reflects the pyroelectric identity of both polarized states of investigated PZT film. Asymmetry of the "tails" of the $\left|U_{\pi 1,2}\left(V_{d c}\right)\right|-$ "wings" and inequality $V_{c}^{-}<V_{c}^{+}$indicates the difference on the earlier stage of repolarization processes.

The shapes of obtained $U_{\pi 1,2}-V$-loops and inequality $V_{c}{ }^{-}<V_{c}{ }^{+}$in general are similar to that known for $P$ - $V$-loops of metal/PZT-film/Si-substrate system [3, 4, 7, 9, 13].

The analysis of $U_{\pi 1^{-}}-V$-loops and $U_{\pi 2^{-}} V$-loops shows earlier and more complete saturation of $U_{\pi 1}\left(V_{d c}\right)$ poling curves comparatively with undersaturated $U_{\pi 2}\left(V_{d c}\right)$ poling curves for the both of $V_{d c}$ polarities. Since $U_{\pi 1} \propto \gamma$ and $U_{\pi 2} \propto \gamma / \varepsilon$, we can conclude that the saturation of $\gamma$ value starts just before $V_{c}^{ \pm}$and finishes just after $V_{c}^{ \pm}$, which is earlier than that of $\gamma / \varepsilon$ value. So this is the $\varepsilon$ value which remains undersaturated under $V_{d c}>V_{c}^{ \pm}$.

The behavior of dielectric ratio $D_{\pi}\left(V_{d c}\right) \propto \varepsilon_{\pi}\left(V_{d c}\right)$ is reflected by $D_{\pi^{-}} V$-loop presented in Figure $2 c$. Observed asymmetry of $D_{\pi^{-}} V$-loop corresponds to the same of the $U_{\pi 1,2}\left(V_{d c}\right)$-loops.

The general view of obtained $D_{\pi}\left(V_{d c}\right)$ changes under poling-repoling cycle reflects the general tendency of decreasing $\varepsilon_{\pi}$ value in the poling run under $V_{d c}$ increase and conservation of lower value of $\varepsilon_{\pi}$ under subsequent decrease of $V_{d c}$. Also the step-like jumps of $D_{\pi}\left(V_{d c}\right)$ in vicinity of $V_{d c}=0$ with the maxima around of $V_{d c}=0$ are observed.

The sharp peculiarities of $D_{\pi}\left(V_{d c}\right)$ around of $V_{d c}=V_{c}$ are visible and the first one at $V_{c}^{+}$is more pronounced than the second one at $V_{c}^{-}$. These peculiarities of $D_{\pi}\left(V_{d}\right)$ reflect the variations of $\varepsilon_{\pi}\left(V_{d}\right)$ and so the states of the domain structure of PZT film with the near zero unipolarity degree.

The behaviour of $\varepsilon_{\pi}\left(V_{d c}\right)$ in the vicinity of $V_{d c}=0$ and $V_{d c}=V_{c}^{ \pm}$can be connected with the peculiarities of rearrangement of domain structure of PZT film which consists in mutual transformations of interconnected $180^{\circ}$ and non- $180^{\circ}$ - (in particular, $90^{\circ}$-) domain groups $[5,14]$.

\subsubsection{Pyroelectric response - time repolarization transient curves}

Figure 3 presents the changes of pyroelectric response amplitude and phase in the course of repolarization of Pt/PZT film/Pt:Ti structure by application of d.c. voltage $V_{d c}$ less and near the coercive ones.

Under application of fixed external repoling d.c. voltage $V_{d c}$ the decrease of $U_{\pi}$ value up to zero followed by subsequent increase of $U_{\pi}$ value up to saturated one is observed. This saturated value remains during a long period after $V_{d c}$ getting off.

Observed behaviour of $\varphi_{\pi}(t)$, namely $180^{\circ}$-change under transition of $U_{\pi}(t)$ through its zero value, corresponds to the change of the sign of pyroelectric reaction due to polarization reversal in time induced by $V_{d c}$ application.

For positive and for negative $V_{d c}$ the similar tendency in the $U_{\pi}(t)$ behaviour but at different time scales is observed. So the strong asymmetry in the time scale of repolarization process development is clearly evident.

Figure 4 presents repolarization transient curves $U_{\pi}(t)$ combined from amplitude and phase time dependences from Figure 3.

For positive and for negative poling voltages the regions of $U_{\pi}(t) \sim U_{\pi}(0)(1-\eta \log (t / \tau))$ are observed and the slope $\eta$ increase with $V_{d c}$ value increase. For negative $V_{d c}$ on the both sides from zero response time the view of $U_{\pi}(t)$ is different at low $V_{d c}$ and similar at high $V_{d c}$. So under negative $V_{d c}$ the characteristics of domain walls motion before and after repolarization are different at low $V_{d c}$ values and are similar at high $V_{d c}$ values. For positive $V_{d c}$ on the both sides from zero response time the slopes of $U_{\pi}(t)$ are different at both low and high $V_{d c}$. So, under positive $V_{d c}$ the characteristics of domain walls motion before and after repolarization are different at low and high $V_{d c}$ values.

In Figure 5 the voltage dependences of zero-response time $t_{0}$ and saturation time $t_{s}$ are presented.

Increase of negative $V_{d c}$ value leads to strong decrease of $t_{s}$ and $t_{0}$ values, which remain near constant when $V_{d c}$ value reaches and exceeds the $V_{c}^{-}$value. Under positive $V_{d c}$ value the same tendency of $t_{0}$ changing but in more narrow time scale is observed. At low $V_{d c}$ the values of $t_{0}$ are 1-2 orders of value higher at negative $V_{d c}$ than at positive ones but at high $V_{d c}$ for the both polarities the values of to are near the same. Under increase of negative $V_{d c}$ the $t_{s}$ value decreases similar to $t_{0}$ but under increase of positive $V_{d c}$ the $t_{s}$ value noticeably increases. So, for negative $V_{d c}$ the similar tendencies and for positive $V_{d c}$ the different tendencies in the behaviour of $t_{0}$ and $t_{s}$ are observed.

\subsection{Dynamic unipolar current-voltage characteristics}

Figure 6 presents the set of dynamic unipolar currentvoltage characteristics obtained under different amplitudes and polarities of drive voltage. 

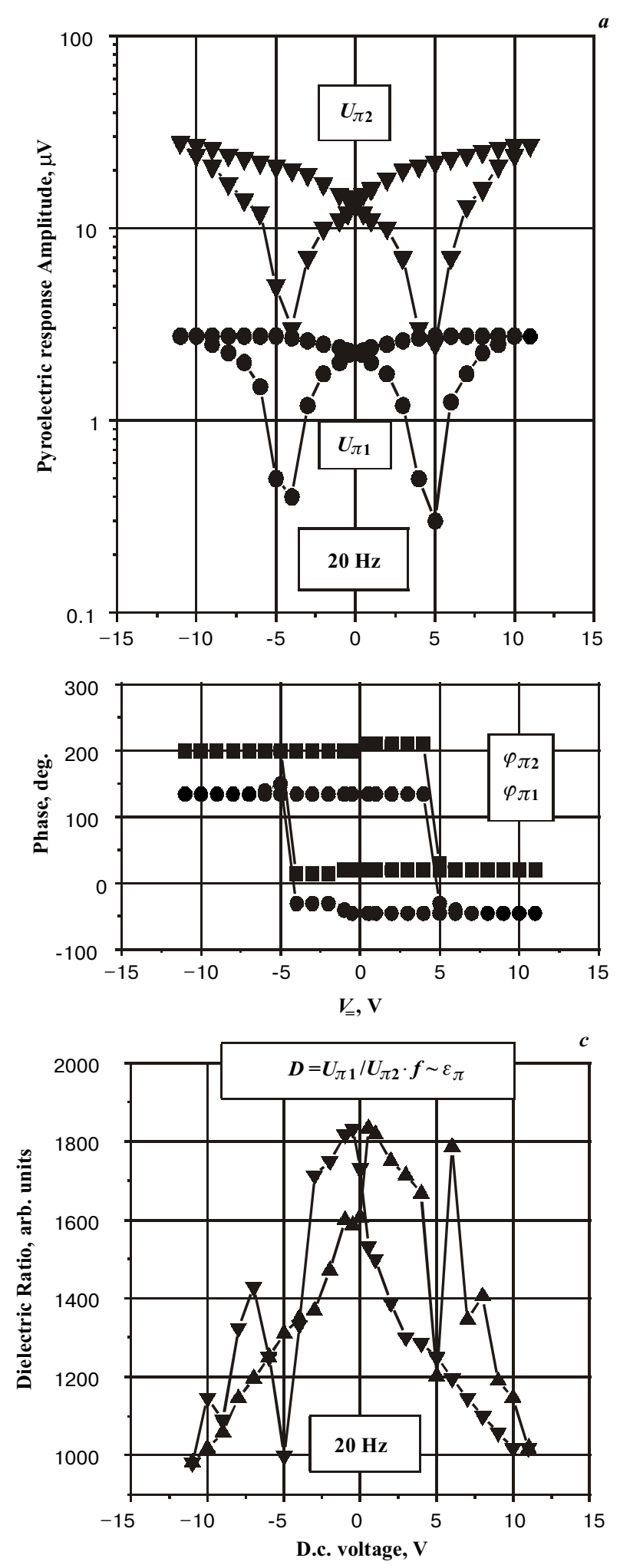

Fig. 2. Pyroelectric response - d.c. voltage loops:

$a$ - "butterflies" of amplitudes $U_{\pi 1,2}(V)$, and parallelograms of phases 1,2(V);

$b$ - hysteresis loops of responses $U_{\pi 2}(V)$ (top) and $U_{\pi 1}(V)$ (bottom);

$c$ - dielectric ratio $D_{\pi}(V)$ loop.
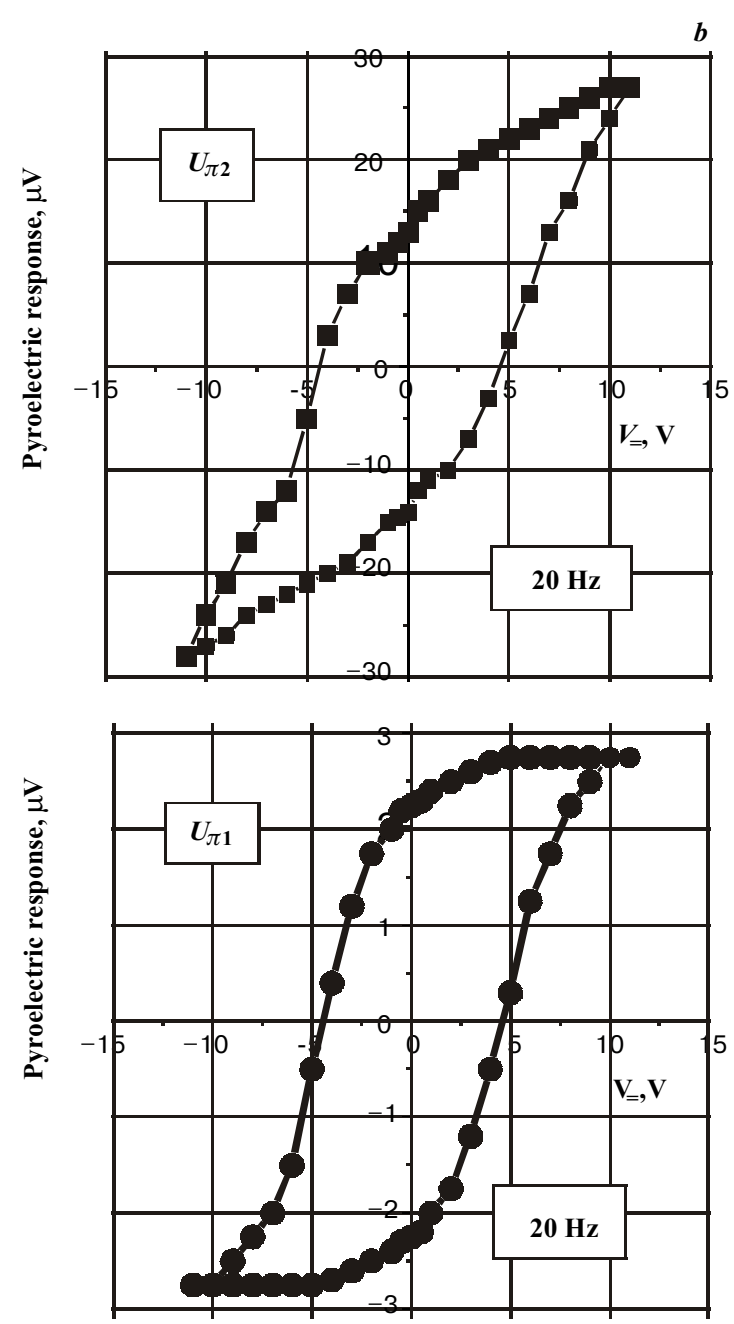

In general, the structure of considered dynamic I-Vcurves is similar to those quasistatic ones observed for many other electroded PZT-film based structures obtained by different technologies [15-17]. On the I-V-curves obtained at $V_{d}<V_{c}$ can be distinguished three characteristic regions. The first one at low $V_{d}$ values is short and jump-like and is connected with displacement current in the moment of $V_{d}$ rise. The second one at intermediate $V_{d}$ values is sub-linear. The third one at high $V_{d}$ values is super-linear.

The voltages $V_{t}^{-}$and $V_{t}^{+}$of transition between sublinear and super-linear regions increase with increasing $V_{d}$ amplitude.

Comparison of the data in Figure 6 shows that the differences of positive and negative branches which increase with the rise of $V_{d}$ values are rather high at short times of $V_{d}$ application and decrease after forming under long term repetitive action of $V_{d}$ saw-pulses. So observed dynamic I-V-curves shows a pronounced dynamic character of polar asymmetry.

For unformed I-V-curves the development of superlinear region with the rise of $V_{d}$ is characteristic. The forming during several minutes leads to the changes of 


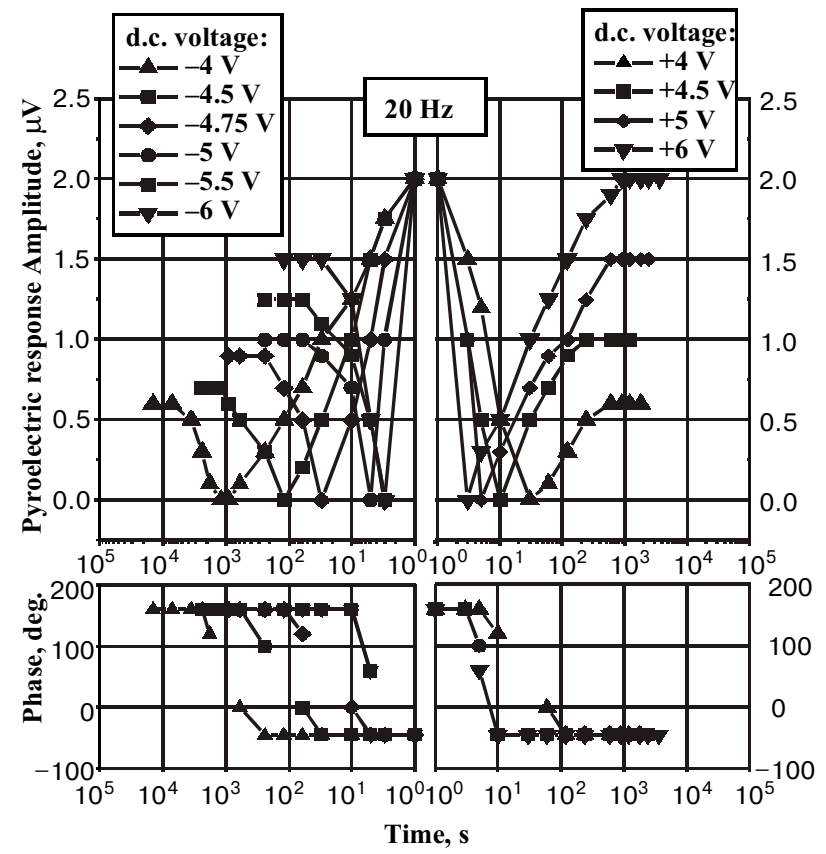

Fig. 3. Pyroelectric response amplitude-time and phase-time repolarization curves: top - pyroelectric response amplitude; bottom - pyroelectric response phase; left - for negative d. c. voltages; right - for positive d. c. voltages.

parameters of the I-V-curves. These changes are more significant under increasing the drive voltage value (compare the data in Figure 6). The expansion of sub-linear region at the expense of super-linear one are observed. Under $V_{d}$ increase the slope of sub-linear region decreases. Under $V_{d}>V_{c}$ the appearance of region with the diffuse maximum and subsequent negative slope is characteristic and more pronounced for the positive branch. These regions are connected with currents of polariza- tion reversal in the definite group of domains ("stubborn domains") which spontaneously return in the initial state under $V_{d}=0$. Taking into account the complex character of mixed $90^{\circ}$ - and $180^{\circ}$ - domain structure in PZT films [5] and the data [14] concerning the contribution of $180^{\circ}$ and non- $180^{\circ}$-domain groups into remanent polarization in PZT films we can suppose that the stubborn domain group in a significant degree consists of non- $180^{\circ}$-domains.

\section{Discussion}

The obtained results show that the investigated systems possess polar asymmetry of pyroelectric parameters, poling time and current-voltage characteristics, which correspond to coexistence of interrelated ferroelectric asymmetry and asymmetry of charge transport.

Indeed, processes of polarization reversal in the $\mathrm{Pt} / \mathrm{PZT} / \mathrm{Pt}: \mathrm{Ti} / \mathrm{TiO}_{2} / \mathrm{SiO}_{2} / \mathrm{Si}$ structures are developed in the condition of asymmetry of injection properties of Pttop and Pt:Ti-bottom contacts due to the difference in the conditions of their manufacturing. Besides, there is an asymmetry of profile of the space charge connected with the profile of mobile and immobile point and expanded defects. In PZT films these are $V_{\mathrm{Pb}}{ }^{+}$and $V_{\mathrm{O}}{ }^{-}$vacancies, domain walls, boundaries of separate micro-regions of PZT film, and also various types of distortions in underelectrode regions of the film.

In the case of investigated Pt/PZT/Pt:Ti structures, because of the complex configuration of the space charge, the charge transport on the different stages of repolarization process can be realized by so called relayrace alternation of different mechanisms (see their list in Ref [17]). The complex structure of observed I-V-curves and its changes in time are in accordance with this interpretation.

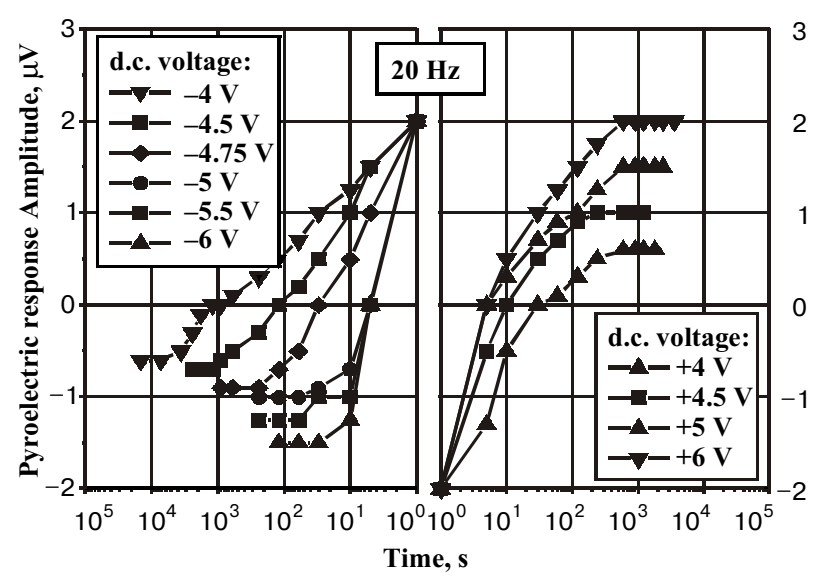

Fig. 4. Pyroelectric response signal - time repolarization transient curves: left - for negative d. c. voltages; right - for positive d. c. voltages.

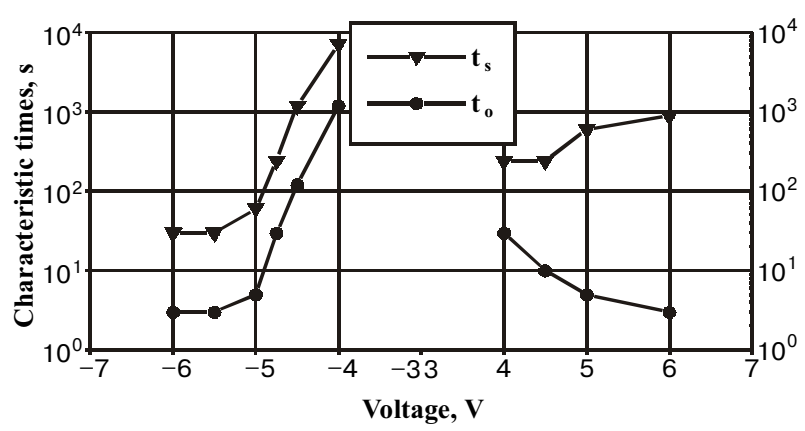

Fig. 5. Voltage dependences of pyroelectric response characteristic times under d. c. voltage repolarization: left - for negative d. c. voltages: right - for positive d. c. voltages. 
S.L. Bravina et al.: Peculiarities and asymmetry of polarization reversal in ...
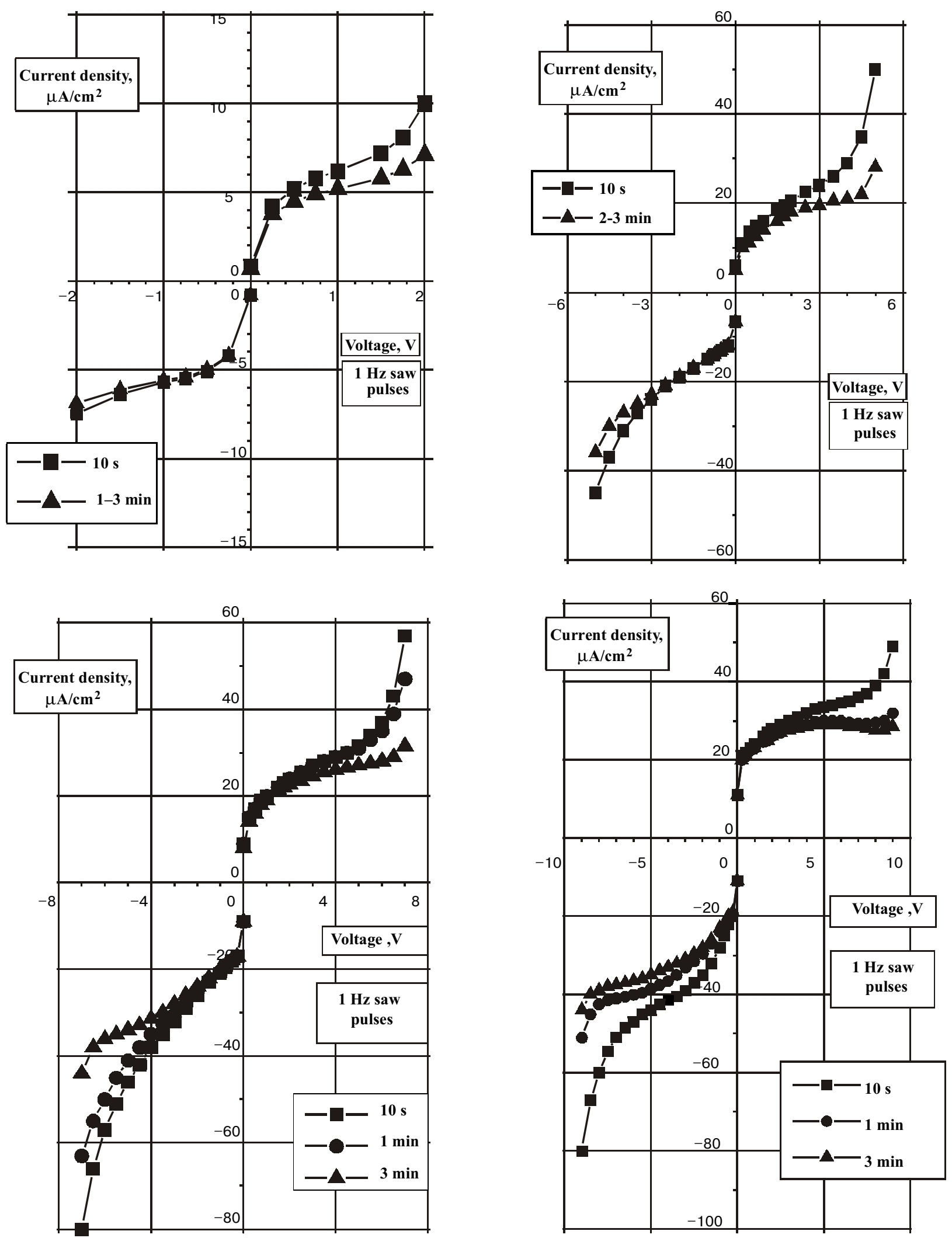

Fig. 6. Dynamic unipolar current-voltage characteristics under different drive voltage amplitudes. 


\section{S.L. Bravina et al.: Peculiarities and asymmetry of polarization reversal in ...}

A super-linear region of $\mathrm{I}-\mathrm{V}$ curves is usually associated with enrichment with charge carriers of near-contact region with metal and discussed in terms of injection mechanisms connected with the existence of currents limited by space charge [18], Schottky emission [19] and Pool-Frenkel ionization [20]. A sub-linear region of I-Vcurves can be connected with depletion of contacting regions of essentially different degree of doping due to exclusion and extraction of charge carriers [21]). The formation of such structure is characteristic for the case of diffusion of metal in semiconductor in the region of their contact as the result of thermal treatment and is quite possible in the course of manufacturing the investigated $\mathrm{Pt} / \mathrm{PZT} / \mathrm{Pt}$ :Ti structures.

Dynamic character of observed current asymmetry points to the change of conditions of charge transport with time. So, the observed pyroelectric asymmetry is developed in the conditions of changing the space charge profile with time.

The changes of pyroelectric response under reversing polarization are connected with domain structure reconstruction. That is why the time of such changes is determined in a high degree with processes of nucleation of new domains and displacement of domain walls.

The peculiarities of domain structure behaviour under polarization direction reversal in the perovskite crystalline ferroelectrics [22] and in PZT films [5, 14, 23] are similar in many details. For initially non-polarized or depolarized crystals and PZT films, contained a number of disordered domains of non- $180^{\circ}$ - (in particular, $90^{\circ}$-) and $180^{\circ}$ - orientation the polarization process occurs under action of external voltage by simultaneous displacement of non- $180^{\circ}$ - and $180^{\circ}$ - domain boundaries, and reorientation of $180^{\circ}$-domains. The process of polarization reversal in PZT films starts from the nucleation and growth of opposite domains (OD) of wedge-like shape from the surface into the depth of the film and finishes by broadening the domains spreading through the film by side displacement of domain walls (DW).

The role of processes of charge transport under polarization reversal in thin layer structures of metalferroelectric-metal type by means of formation OD and displacement of charged DW was considered earlier in $[24,25]$. Facilitation of the conditions of domain nucleation and growth in film structures due to presence of dielectric non-uniformity was considered recently in [26, 27]. To provide the displacement of charged DW it is necessary to supply the certain value of mobile electric charges, which is necessary for compensation of changeable bounded charge. The peculiarities of this DW displacement define the peculiarities of $U_{\pi^{-}} V$ - and $U_{\pi^{-}} t$ curves.

Under registration of $U_{\pi^{-}} V$ - poling curves the increase of $U_{\pi}\left(V_{d c}\right)$ from zero value to the maximal one at given $V_{d c}$ value starts in the system of mutually pyroelectrically compensated OD and occurs in the system of rearranged OD. At that the value $V_{d c}=V_{t h}$ corresponds to the beginning of sharp change of $U_{\pi}$ under $V_{d c}$ increase and so the start of fast rearrangement in the system of OD.
Under registration of $U_{\pi^{-} t \text {-curves of repolarization }}$ the variation of $U_{\pi}(t)$ reflects the trend of compensation process in the preliminary arranged OD system. The moment $t=t_{0}$ when $U_{\pi}\left(t_{0}\right)=0$ corresponds to pyroelectrically compensated state of OD system. So the time $t=t_{0}$ corresponds to transport of such value of electrical charge, which is necessary for creation of number of OD required for compensation.

The fact of vanishing $U_{\pi}$ values at $V_{d}=V_{c}{ }^{ \pm}$under $U_{\pi^{-}}$ $V$-loops cycling and also at $t=t_{0}{ }^{ \pm}$under $U_{\pi^{-} t \text {-curves re- }}$ gistration permits to consider some analogies between the coercive voltages $V_{c}{ }^{ \pm}$and zero response times $t_{0}{ }^{ \pm}$.

Starting from the definition of the effective degree of unipolarity $\xi=\left(c^{+}-c^{-}\right) /\left(c^{+}+c^{-}\right)$, where $c^{+}$and $c^{-}$are the concentrations of the domains with the opposite directions of the components of polarization vector $P_{S}$ along the thickness of the film, we can conclude that at $V_{d}=V_{c}^{ \pm}$ and at $t=t_{0}{ }^{ \pm}$the value $\xi=0$. Since the values $V_{d}=V_{c}^{ \pm}$ and $t=t_{0} \pm$ correspond to the similar states of the domain structure, namely those just balanced between two inversely poled states, we can consider $t_{0}$ as the coercive times. Indeed, from the standpoint of transport of charge value necessary for vanishing the value of integral polarization, the coercive voltage is the necessary voltage value under given time of its action, and the coercive time is the necessary time under given voltage value.

\section{Conclusions}

The performed investigation has shown the strong correlation between the poling pyroelectric and so ferroelectric and electrical asymmetries.

Observed behaviour of $U_{\pi^{-}} V$ - and $U_{\pi^{-t}}$-curves and also $U_{\pi^{-}} V$-loops displays the considerable polar and time asymmetry of polarization reversal.

Observed transformation of I-V-curves is the evidence of considerable $V$ - and $t$-dependent variation of charge transport conditions.

Changes of pinning conditions, domain wall motion characteristics, charge and mechanical state of the point (immobile and mobile) and extended (domain wall) defects is the reason of observed complex asymmetry.

For the investigated PZT thin films on Si systems with complex composition and asymmetric space charge profile is characteristic its change with time under the action of applied external voltage.

The consequence of contact asymmetry and space charge asymmetry is the difference in the conditions of electric charge transport under the different polarity of applied voltage. It results, in particular, in different time of space charge accumulation necessary for reversing the polarization direction and also in different spread of this process in time. It leads to the difference in coercive times

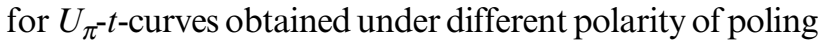
voltage, and also to the difference in coercive voltage values obtained for $U_{\pi 1^{-}} V$ - and $U_{\pi 2^{-}} V$-loops under different polarity of drive voltage.

For as more complete as possible examination of polarization reversal processes in PZT thin films on Si struc- 


\section{S.L. Bravina et al.: Peculiarities and asymmetry of polarization reversal in ...}

tures it is necessary to perform the investigation of the complex of their electrophysical characteristics including not only ferroelectric but at the least pyroelectric and current-voltage characteristics.

\section{Acknowledgements}

Authors gratefully acknowledge Ministry of Science of France and the University of Valenciennes for financial support.

\section{References}

1. B. Jaffe, W. R. Cook, H. Jaffe, Piezoelectric ceramics, Acad. Press., London, 1971.

2. L.S. Kremenchugsky and O.V. Roitsina, Pyroelectric Detectors of Radiation, Naukova Dumka, Kiev, 1979 (in Russian).

3. Y. Ishibashi, Ferroelectric Thin Films: Synthesis and Basic Properties, Gordon and Breach, Amsterdam, pp. 135-152, 1996.

4. V.Y. Shur, Ferroelectric Thin Films: Synthesis and Basic Properties, Gordon and Breach, Amsterdam, pp. 153-169, 1996

5. R. Waser, Modeling of electroceramics - application and prospects // J. Europ. Ceram. Soc., 19, pp. 655-664 (1999).

6. Y. Xu, C. J. Chen, R. Xu, J. D. Mackenzie, Self biased heterojunction effect of ferroelectric thin film on silicon // Ferroelectrics, 108(1-4), pp. 47-52 (1990).

7. W. Liu, J. Ko, W. Zhu, Asymmetric switching behavior of Ni/ $\mathrm{Pb}_{1,1}\left(\mathrm{Zr}_{0,3} \mathrm{Ti}_{0,7}\right) \mathrm{O}_{3} / \mathrm{Pt}$ thin films // Material Letters, 49, p. 122 (2001).

8. S.L. Bravina, N.V. Morozovsky, A.A. Strokach, Pyroelectricity: Some Physical and Application Aspects // Proceedings of SPIE, 3182, pp. 85-99 (1997).

9. G. Velu, D. Remiens and B. Tierry, Ferroelectric properties of PZT thin films prepared by sputtering with stoichiometric single oxide target: comparison between conventional and rapid thermal annealing // J. Europ. Ceram. Soc., 17, p. 1749 (1997).

10. T. Haccart, E. Kattan, D. Remiens, Dielectric, ferroelectric and piezoelectric properties of sputtered PZT thin films on $\mathrm{Si}$ substrates: influence of film thickness and orientation // Semicond. Phys., Quant. Electron. and Optoelectronics, 5(1), pp. 78-88 (2002).

11. N.V. Morozovsky, V.B. Samoilov, I.A. Stoyanov, Investigation of matching stages for pyroelectric detectors of radiation, in book Thermal Detectors of Radiation, GOI, Leningrad, pp. 104-105 (1980).
12. S.L. Bravina, L.S. Kremenchugsky, N.V. Morozovsky et.al., Investigation of Phase Transitions in $\mathrm{Ag}_{3} \mathrm{AsS}_{3}$ and $\mathrm{Ag}_{3} \mathrm{SbS}_{3}$ by Method of Dynamic Pyroelectric Effect, Preprint No. 26, Inst. of Phys. of Acad. Sci. of Ukraine, Kiev, 1982 (in Russian).

13. P. Pertsch, M.-J. Pan, V. R. Vedula, S. Yoshikawa, S.-E. Park, T.R. Shrout, Characteristics of electromechanical solid state multilayer actuators // IEEE, pp. 571-574 (1998).

14. K. Saito, T. Oikawa, T. Kurosawa, T. Akai and H. Funakubo, Role of non-180o domain switching in electrical properties of $\mathrm{Pb}(\mathrm{Zr0} 0.35 \mathrm{Ti0} .65) \mathrm{O} 3$ thin films // Jpn. J. Appl. Phys., 41, Part 1, No 11B, pp. 6730-6734 (2002).

15. H. Miki, K. Kushida-Abdelghafar, K. Torii, Y. Fujisaki, Hydrogen related degradation and recovery phenomena in $\mathrm{Pb}(\mathrm{Zr}, \mathrm{Ti}) \mathrm{O} 3$ capacitors with a platinum electrode // Jpn. J. Appl. Phys., 36, Part 1, No. 3A, pp. 1132-1135 (1997).

16. M. Brazier, M. McElfresh, S. Mansour, Origin of anomalous polarization offsets in compositionally graded $\mathrm{Pb}(\mathrm{Zr}, \mathrm{Ti}) \mathrm{O}_{3}$ thin films // Appl. Phys. Lett., 74(2), pp. 299-31 (1999).

17. Stolichnov and A. Tagantsev, Space-charge influencedingection model for conduction in $\mathrm{Pb}\left(\mathrm{Zr}_{\mathrm{x}} \mathrm{Ti}_{1-\mathrm{x}}\right) \mathrm{O}_{3}$ thin films // J. Appl. Phys., 84(6), pp. 3216-3225 (1998).

18. M. A. Lampert and P. Mark, Current Injection in Solids, Academic, New-York, 1970.

19. W. Monch ed., Electronic Structureof Metal-Semiconductor Contacts, Kluwer Academic, Dordrecht, 1990.

20. F.F. Volkenshtein, Electrical Conductivity of Semiconductors, Techteorizdat, Moscow-Leningrad, 1947 (in Russian).

21. V.L. Bonch-Brujevitch, S.G. Kalashnikov, Physics of Semiconductors, Nauka, Moscow, 1977.

22. E.G. Fesenko, V.G. Gavriliatshenko, A.F. Sementshev, Domain structure of multi-axes ferroelectrics, Ed. of Rostov. Univ., Rostov-on-Don, 1990 (in Russian).

23. D. Fu, K. Suzuki, K. Kato, M. Minakata, H. Suzuky, Investigztion of domain switching and retention in orientated $\mathrm{PbZr}_{0,3} \mathrm{Ti}_{0,7} \mathrm{O}_{3}$ thin film by scanning force microscopy // Jpn. J. Appl. Phys., 41, Part 2, No. 11B, pp. 6724-6729 (2002).

24. G.M. Guro, I.I. Ivanchik, N.F. Kovtoniuk, C-Domain Crystal $\mathrm{BaTiO}_{3}$ in the Short Circuited Capacitor // Sov. Sol. St. Phys., 11(7), pp. 1956-1964 (1969).

25. V. F. Krapivin and E. V. Chensky, Space Charge Limited Currents in the system Metal-Ferroelectric-Metal // Sov. Sol. St. Phys., 12(2), pp. 597-604 (1970).

26. A. M. Bratcovsky, A. P. Levaniuk, Abrupt appearance of the pattern and fatigue of thin ferroelectric films // Phys. Rev. Lett., 84(14), pp. 3177-3180 (2000).

27. A. M. Bratcovsky, A. P. Levaniuk, Ease polarization switching in ferroelectrics // Phys. Rev. Lett., 8(1), pp. 4614-4617 (2000). 